\title{
Liver Metastatic Colorectal Tumor Cells Change Their Phenotype During Consecutive Passages on Chick Embryo Chorioallantoic Membrane: Lessons from the Lab to the Clinic
}

\author{
RALUCA AMALIA CEAUȘU ${ }^{1,2}$, ALEXANDRU CIOLOFAN $^{3,4}$, ALEXANDRU BLIDIȘEL $^{3,4}$, \\ OCTAVIAN MARIUS CREȚU ${ }^{3,4}$, ANCA MARIA CIMPEAN ${ }^{1,2}$ and MARIUS RAICA ${ }^{1,2}$ \\ ${ }^{1}$ Department of Microscopic Morphology/Histology, \\ Victor Babes University of Medicine and Pharmacy, Timisoara, Romania; \\ ${ }^{2}$ Angiogenesis Research Center, Victor Babes University of Medicine and Pharmacy, Timisoara, Romania; \\ ${ }^{3}$ Department IX/Surgery I, Victor Babes University of Medicine and Pharmacy, Timisoara, Romania; \\ ${ }^{4}$ Center for Liver, Gall Bladder and Pancreas Surgery, Victor Babes \\ University of Medicine and Pharmacy, Timisoara, Romania
}

\begin{abstract}
Background/Aim: Colon cancer liver metastases with desmoplastic growth pattern (dGP) have a highly heterogeneous therapy response. The aim of the study was to evaluate the $d G P$ liver metastasis molecular profile from a chemo-naive patient by mimicking metastatic process on an experimental chick embryo chorioallantoic membrane (CAM) model. Materials and Methods: Three successive CAM passages of dGP human colorectal liver metastases were immunophenotyped for keratin (K) 8, and 20, CLIC1, VEGF, EGFR, CD34, podoplanin, Ki67, E-cadherin and vimentin. Results: Metastatic cells gradually lost K20 while K8, E-cadherin and vimentin heterogeneously increased during passages. VEGF, CLIC 1, EGFR expression increased in metastatic cells especially at the tumor graft periphery. Scattered proliferating and non-proliferating podoplanin-positive tumor cells, lymphatic and blood vessels were heterogeneously detected in tumor xenografts depending on passage stage. Conclusion: By mimicking repetitive metastatic processes we proved that metastatic cells change their phenotype. This may explain why not all metastases have a similar response to therapy.
\end{abstract}

This article is freely accessible online.

Correspondence to: Alexandru Blidisel, MD, Ph.D., Lecturer, Department IX/Surgery I "Victor Babes" University of Medicine and Pharmacy, Department of Surgery, Piata Eftimie Murgu no. 2, 300041, Timisoara, Timis, Romania. Tel: +40 722574552, e-mail: blidy@umft.ro

Key Words: Colon cancer, liver metastasis, chick embryo chorioallantoic membrane, tumor heterogeneity.
Despite advances in therapy, as the use of the resection combined with adjuvant systemic regimens, the use of the portal vein embolization techniques, the new findings in the molecular aspects of the colorectal liver metastasis (CRLM), the curative ratio is only $20 \%$ (1-3). It was shown that perioperative systemic therapy in patients with resecable CRLM before and after curative hepatic resection does not improve the 5-year overall survival (OS) for these patients compared to those treated with hepatic resection alone $(51 \%$ vs. $48 \%)(4,5)$.

The histological growth pattern (HGP) has been noticed in the liver metastasis (LM) with colorectal (CR) origin, but also in those from breast, gastric and uveal melanoma. The HGPs were found in other sites in the lung (pushing, desmoplastic and aerogenous types) and brain (welldemarcated one, vascular co-option and diffuse infiltration types) metastases of CR carcinoma (6-8). The HGP of LM with $\mathrm{CR}$ origin was proposed as a prognosis parameter by an international multidisciplinary team (9). From the main HGP, (desmoplastic, replacement, pushing and mixed histological growth pattern GP) a superior survival rate was noticed in the desmoplastic growth pattern (dHGP) compared to replacement HGP. Analyzing the correlation of HGP with the immune phenotype (IP) and clinical outcome after liver resection, Stremitzer et al. noticed that inflammatory IP was associated with dHGP. The dHGP was associated with better radiological, histological and bevacizumab-based chemotherapy response compared to replacement HGP. dHGP is associated with inflamed IP and HGP may be a potential biomarker for adjuvant treatment that includes targeting the immune microenvironment (10). 
The desmoplastic type of liver metastases is characterized by the fibrotic reaction that surrounds the metastases and angiogenesis with an increased rate of endothelial cell proliferation and microvascular density (11). The chick chorioallantoic membrane (CAM) model is an intensely used experimental model for tumor and cancer cell lines growth because of its low cost and lack of the immune response. The biology of HGPs and the mechanisms of capsule formation in the desmoplastic type are not completely understood.

Based on these data, the aim of this study was to evaluate the molecular profile of liver metastases (desmoplastic type), with colorectal origin in the case of successive transfers on chick chorioallantoic membrane (CAM).

\section{Materials and Methods}

Patients and biopsies. The present study included LM of CR adenocarcinoma biopsy obtained by an excisional tumorectomy of the LM. Signed consent was obtained from the patient, the principles of the Declaration of Helsinki were respected, and the study was approved by the Institutional Review Board (no. $7339 / 22.04 .2016$ ). Some of the metastatic fragments were fixed in $10 \%$ buffered formalin for $24 \mathrm{~h}$ and paraffin embedded for morphological and immunohistochemical evaluation and others were applied on the CAM.

Experimental design. Fertilized white Leghorn chicken eggs were used in this study. The eggs were divided into 2 groups, each group containing 10 eggs, plus the control group and incubated at a temperature of $37^{\circ} \mathrm{C}$, in a humid atmosphere. On the third day of incubation, the eggs were opened, and a window was made on the surface of the egg, later covered with adhesive tape. A silicone ring was applied to the surface of the CAM on the first day of the experiment, which corresponded to the eight day of incubation. In the ring, a suspension of tumor cells derived from CRLM (dHGP) was implanted. After 5 days, the xenograft was transferred to the membrane of another egg. This was followed by the second transfer, after 5 days.

Immunohistochemistry. CLIC 1 (Santa Cruz Biotechnology, Santa Cruz, CA, USA, monoclonal, clone 356.1, dilution 1: 2,000), EGFR (Novocastra Newcastle Ltd, Newcastle Upon Tyne, UK, clone EGFR.113, dilution 1:20), VEGF (Dako, Glostrup, Denmark, DK2600, clone VG1, RTU), K20 (clone PW31), vimentin (clone V9), E cadherin (clone 26B5), Ki67 (clone MM1) were used as primary antibodies. All of the last antibodies were ready to use, from Leica Biosystem, Newcastle Ltd, Newcastle Upon Tyne, UK. Heatinduced epitope retrieval with Bond Epitope Retrieval Solution 2 (Leica Biosystems) for $20 \mathrm{~min}$ was followed by endogenous peroxidase blocking ( $5 \mathrm{~min}$ ), incubation with primary antibodies (20 min) and visualization with The Bond Polymer Refine Detection System (15 min). 3,3-diaminobenzidine dihydrochloride was used as chromogen (10 min) and hematoxylin was used as a counterstain. In the case of double immunostainings (vimentin/keratin 8/18, E-cadherin/vimentin, Ki67/CD34, Ki67/Podoplanin) the previously mentioned steps, without the application of hematoxylin, were followed by: endogenous peroxidase block ( $5 \mathrm{~min}$ ), incubation with secondary antibodies, visualization with Bond Polymer Refine Red
Detection System, Leica Biosystems), 3,3- diaminobenzidine dihydrochloride $(10 \mathrm{~min})$ and hematoxylin as a counterstain for 5 min. The secondary antibodies were ready to use, from Leica Biosystems: keratin 8/18 (monoclonal, clone 5 D3), vimentin (monoclonal, clone V9), CD34 (monoclonal, QBEnd 10) excepting Podoplanin (Abcam plc, Discovery Drive, Cambridge, UK, clone gp36, dilution 1:40). The full immunohistochemical procedure was performed with Bond Max Autostainer (Leica Biosystem).

In the simple and double immunohistochemical stainings, the cases that showed the cytoplasmic expression (CLIC 1, K20, EGFR, VEGF, podoplanin, vimentin, CD34), membrane (E-cadherin) and nuclear (Ki67) were included in the study.

Microscopic evaluation and data analysis. All slides were scanned by the Desk Pannoramic Scanner (3D Histech, Budapest, Hungary) and they were stored on Histology Department Digital Slides Library Case Center). The evaluation of the slides, pictures capture, and processing were performed by using Pannoramic Viewer pathology software (3D Histech).

\section{Results}

Histopathology and liver metastasis immunophenotype before CAM implantation. The morphological evaluation of LM showed the dHGP, with the presence of a connective tissue band at the border of metastatic area and the remaining liver tissue. The CR origin of LM was supported by keratin 20 positivity with granular cytoplasmic pattern. A homogeneous distribution and heterogeneous intensity patterns (with values of 2 and 3) were noticed in the metastatic area. A granular cytoplasmic pattern of EGFR, with distribution and intensity heterogeneity were noticed. EGFR-positive metastatic cells with intensity values between 1 and 3 were found. The CLIC 1 expression pattern was predominantly granular cytoplasmic and nuclear in few, isolated cells. CLIC 1 was found in the blood vessels endothelial cells from connective tissue band, in the liver sinusoidal endothelial cells and in few starry-like morphology cells disposed under the liver capsule. VEGF immunoreaction revealed negative cells and positive cells with intensity values of 2 and 3 in the metastatic area. Positive cells with an intensity value of 3 were present in the connective tissue that delimits the metastatic area from the remaining liver. The double immunostaining D2-40/Ki67 showed the following metastatic cells phenotypes: negative, D2-40 positive, Ki 67 positive and D2-40/Ki 67 positive cells. D2-40 positive lymphatic vessels were noticed under the liver capsule, in the remaining liver parenchyma.

A suspension of cells belonging to a LM of CR origin was applied to the surface of the chick embryo chorioallantoic membrane (CAM) (Figure 1a). After four days, during which the xenografts increased in size, by blood vessel acquisition from the CAM, the transfer was performed on the membrane of another embryonated eggs. Macroscopically, the newly transferred tumor acquired vessels, visible at the periphery, 


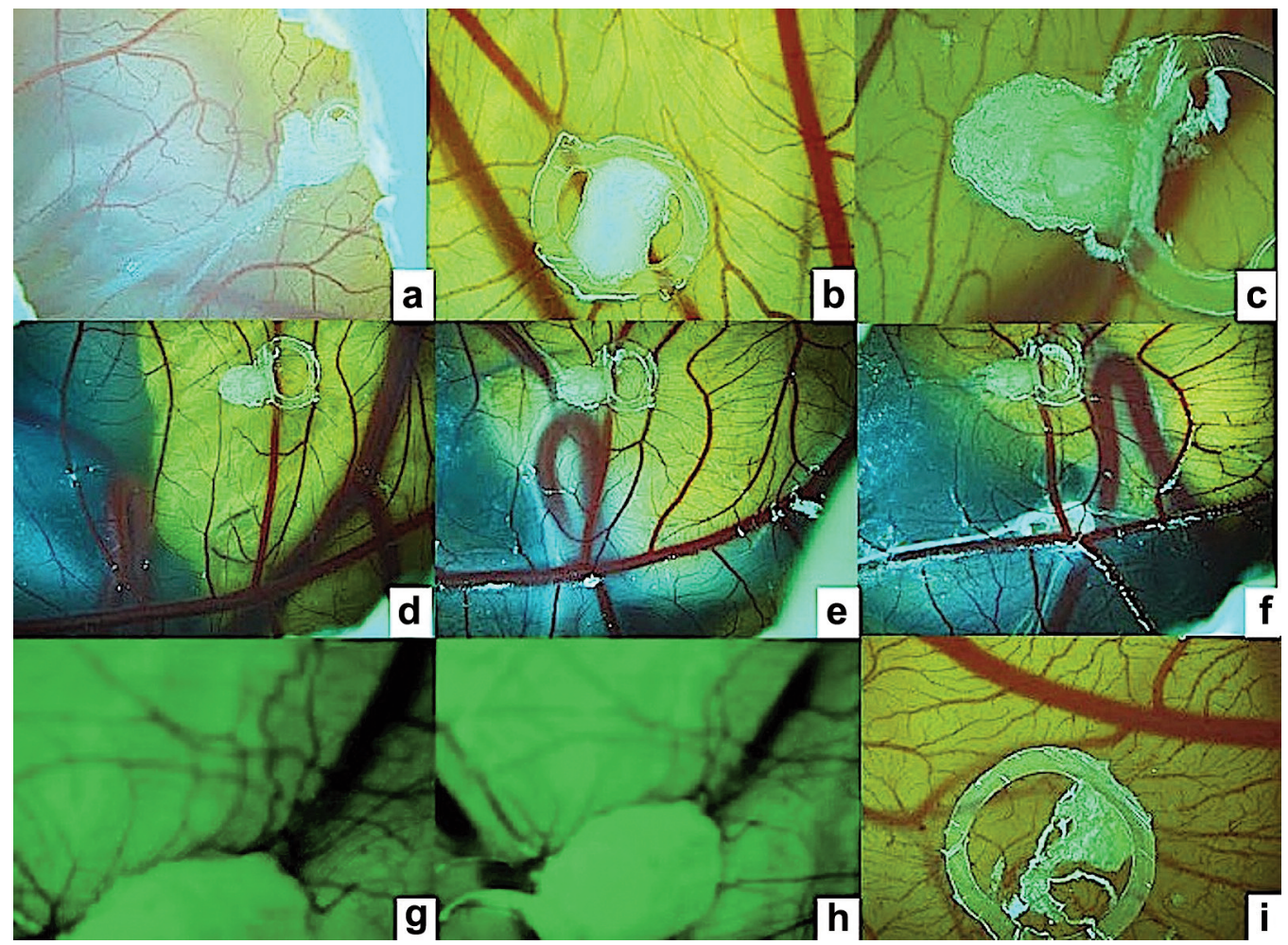

Figure 1. Macroscopic features of xenografts from desmoplastic histological growth pattern (dHGP) of colorectal liver metastasis (CRLM). (a) dHGP of CRLM xenograft initially implanted on chorioallantoic membrane (CAM); dHGP of CRLM on first day (b), second day (c), third day (d), fourth day $(e)$ and fifth day $(f, g)$ after the first transfer; the xenograft on the fifth day after the second transfer $(h, i)$.

with a spoke wheel pattern (Figure 1b-e). The inner vascular network of the graft was visible on the third day after transfer and the graft growth continued (Figure $1 \mathrm{f}$ and g). On the fifth day after the first transfer, the second transfer took place (Figure 1h and i).

Tumor cell immunophenotype of the initial implant on CAM. The immunohistochemical profile of the xenograft before the first transfer revealed EGFR and K20 immuno-expression (Figure $2 \mathrm{~b}$ and c). K20 was positive but with lower intensity and distribution compared to metastasis. CLIC 1 immunoexpression was noticed in more than $70 \%$ of metastatic cells with cytoplasmic pattern, only few cells with nuclear expression were seen (Figure 2a). Reaction intensity values varied between 2 and 3 (isolated cells). VEGF-positive cells with intensity values of 2 and 3, predominantly distributed around the blood vessels were found (Figure 2d).

Tumor cells immunophenotype heterogeneity during consecutive passages on CAM. The morphological staining of the transferred xenograft, on the first day after transfer, indicated the preservation of the initial metastatic profile, of
dHGP, with collagen bands at the periphery of the metastatic areas. A heterogeneous distribution pattern of K20 with negative and positive xenograft cells (intensity value of 3 , more numerous; Figure 2g) was noticed. EGFR was expressed with a heterogenous intensity and distribution pattern in the transferred xenograft than the initial one (intensity values of 2 and 3; Figure 2f). A slight decrease in the number of VEGF-positive cells was noticed, but the intensity and distribution pattern were maintained.

Morphological and immunohistochemical evaluation of the xenograft two days after first transfer indicated a decrease in the immunoreaction intensity and number of cells for keratin 20 compared to a previous evaluation. A decrease in the number of positive cells and the intensity of the reaction for CLIC 1, EGFR, K20, VEGF was found. The distribution of positive cells for all antibodies was predominantly at the periphery of the xenograft (Figure 2i-1).

The xenograft on the fifth day after the second transfer showed a higher percentage and intensity of reaction for CLIC 1 (value 3 of intensity, cytoplasmic and nuclear pattern), EGFR and VEGF-positive cells. An important decrease in the number of positive cells (until no keratin 20 


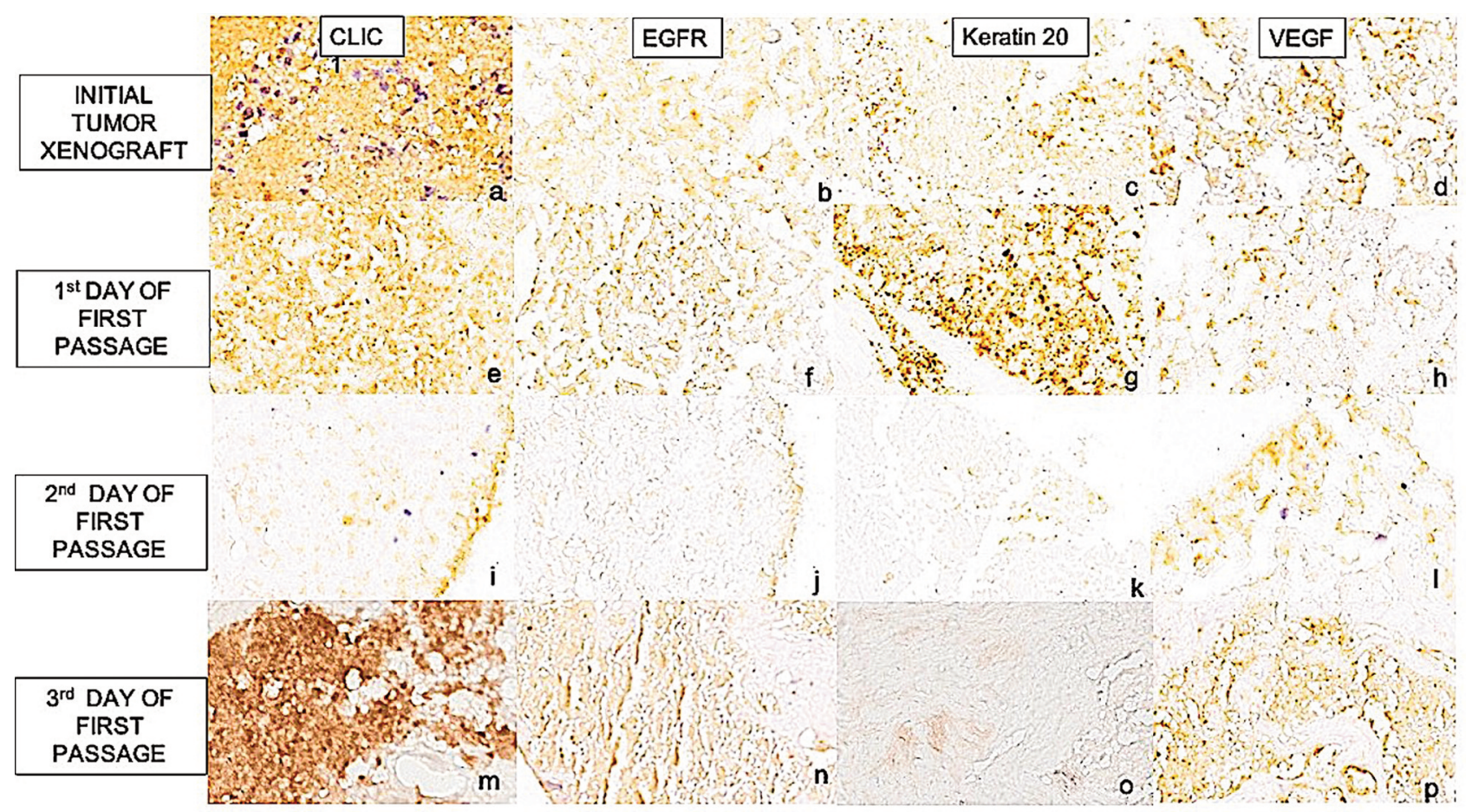

Figure 2. Immunohistochemical expression of chloride intracellular channel protein 1 (CLIC1), epidermal growth factor receptor (EGFR), keratin 20, vascular endothelial growth factor (VEGF) (vertically) before transfer, first day after transfer, second day after first transfer and the fifth day after the second transfer (horizontally).

expression) and immunoexpression intensity in the xenograft cells was noticed (Figure 2m-p).

The main cellular phenotypes highlighted in xenografts by double immunoreactions were: epithelial (E-cadherin ${ }^{+} / \mathrm{vim}^{-}$; $\mathrm{K}^{+} / \mathrm{vim}^{-}$), mesenchymal (E-cadherin ${ }^{-} / \mathrm{vim}^{+} ; \mathrm{K}^{-} / \mathrm{vim}^{+}$), mesenchymal-epithelial or non-differentiated phenotype $\left(\right.$ E-cadherin $\left.{ }^{+} / \mathrm{vim}^{+} ; \mathrm{K}^{+} / \mathrm{vim}^{+}\right)$and differentiated phenotype $\left(\right.$ E-cadherin ${ }^{+} / \mathrm{K}^{+}{ }^{+}$).

On the first day after the first transfer the xenograft was characterized by the predominance of the epithelial phenotype in the metastatic cells and epithelial- mesenchymal differentiated phenotype. Only few cells with mesenchymal and mesenchymal non-differentiated phenotype were noticed (Figure 3a-c).

On the second day after the second transfer, a decrease of value intensity reaction for E-cadherin and the number of epithelial phenotype cells (E-cadherin ${ }^{+} / \mathrm{vim}^{-} ; \mathrm{K}^{+} / \mathrm{vim}^{-}$) (Figure $3 \mathrm{~d}$ and $\mathrm{f}$ ) and a slight increase in the number of mesenchymalepithelial differentiated phenotype (Figure $3 \mathrm{e}$ and $\mathrm{f}$ ). Isolated cells with mesenchymal and mesenchymal-epithelial nondifferentiated phenotypes were noticed (Figure 3d-f).

On the fifth day after the second transfer a higher value of $\mathrm{K} 8$ intensity in epithelial phenotype cells was noticed (Figure $3 \mathrm{~h}$ and i). Isolated cells, with mesenchymal phenotype was also found (Figure $3 g$ and $\mathrm{i}$ ).
The double immunostaining CD34/ Ki67 revealed vessels with lumen, with CD34-positive endothelial cells and cordlike structures with CD34-positive cells in the peripheral band of the xenograft on the first day after the transfer (Figure $4 \mathrm{a}$ and $b$ ). In the inner part of the xenograft, cord-like structures containing CD34/ Ki67-positive cells, Ki67- positive cells and CD34- positive cells were noticed (Figure 4c).

On the second day after the first transfer, in the connective band from the xenograft periphery, cord like structure consisting of CD34/Ki67-positive cells and CD34-positive cells were noticed. Small groups of CD34, Ki67- positive and co-expressing cells and permeable blood vessels, with lumen and proliferative endothelial cells were present in the peripheral connective tissue band (Figure 4e). Inside of the xenograft, clusters of CD34, Ki67- positive cells and coexpressing cells centered by proliferative, permeable vessels were found (Figure $4 \mathrm{f}$ and $\mathrm{g}$ ). The cord-like structure and CD34-isolated positive cells were found also.

On the fifth day after the second transfer in the peripheral collagen rim the following structures were noticed: cordslike structures consisting of CD34/Ki67-positive cells, network of cords-like structures, vessels with large lumen and proliferative endothelium and vessels with large lumen, pillars inside and proliferative endothelium. All these aspects 


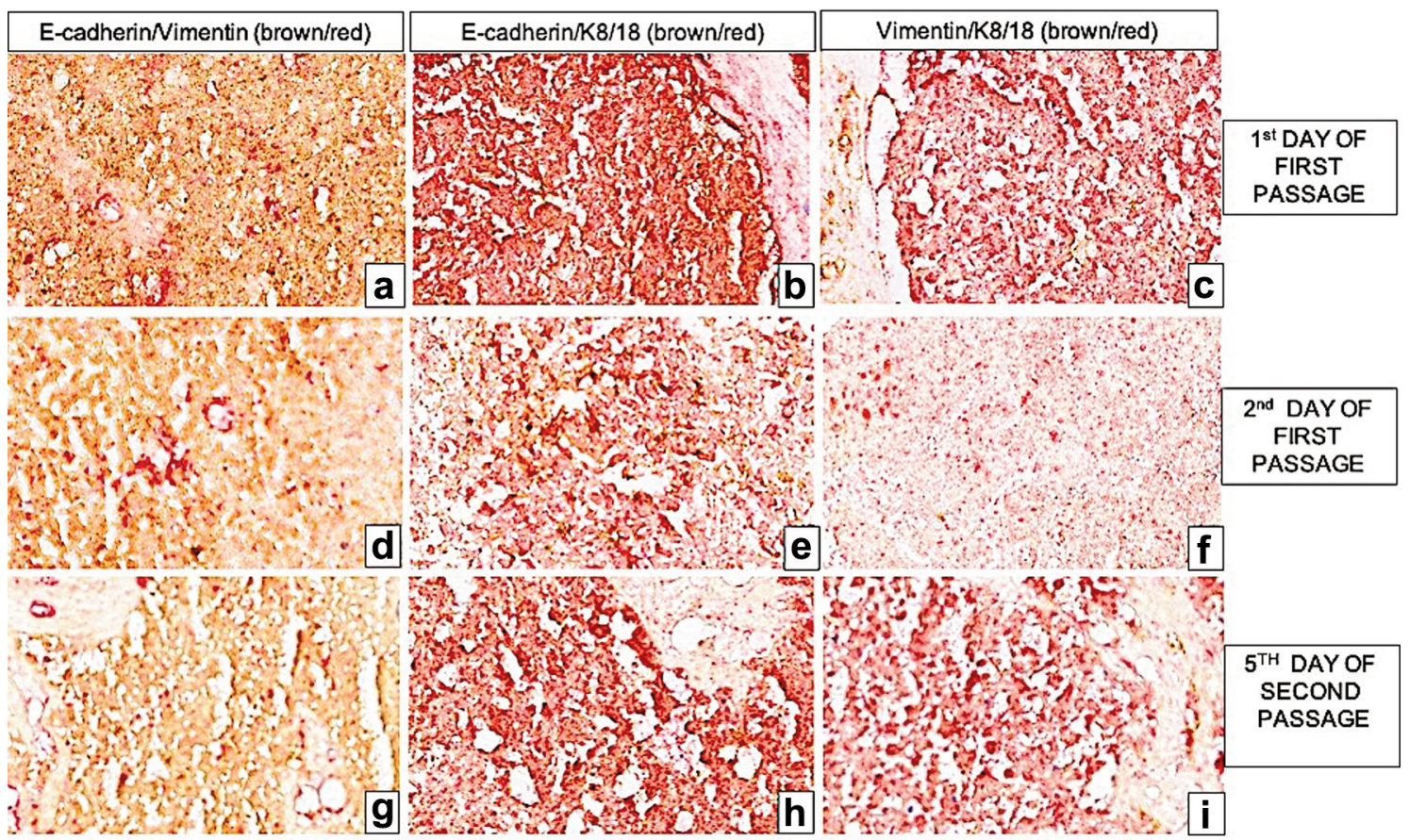

Figure 3. Immunohistochemical expression of E-cadherin/vimentin (brown/red), E cadherin/Keratin 8/18 (k8/18) (brown/red), vimentin/k8/18 (brown/red) (vertically) first day after transfer, second day after first transfer and the fifth day after the second transfer (horizontally).

support two types of angiogenesis: sprouting and intussusception (Figure $4 \mathrm{i}$ and $\mathrm{j}$ ). In the inner part of the xenograft small groups of CD34 and CD34/Ki67-positive cells were found. The main mechanism of angiogenesis within the xenograft was that of intussusception (Figure 4k).

In the xenograft before transfer, groups of podoplaninpositive cells with cytoplasmic expression pattern and intensity values of 1 and 2 were present. Small groups of coexpressing podoplanin/Ki 67 in the metastatic area and in the peripheral connective tissue band were found. Podoplaninpositive vessels were noticed in the metastatic area and collagen band.

On the first day after the first transfer, few lymphatic vessels with narrow lumen were noticed. Isolated vessels with proliferative endothelial cells (podoplanin/Ki67positive) were found in the connective tissue band. Small groups of podoplanin/Ki67- positive cells were detected in the periphery (collagen band) but inside of the xenograft also (Figure 4d).

The xenograft on the second day after the first transfer was characterized by the absence of the expression in the peripheral connective rim and the presence by few, isolated D2-40/Ki67-co-expressing cells inside of the xenograft (Figure 4h).

The features of the xenograft after the second transfer consisted the presence of a higher number of vessels than in the first transfer, first day, identified in the peripheral connective band, but also in the central area of the xenograft. Their lumen was covered by podoplanin-positive endothelial cells or by proliferative lymphatic endothelial cells (podoplanin/Ki67-positive cells). In the inner and peripheral area of the xenograft, groups of podoplanin, Ki67 and podoplanin/Ki67-positive cells were noticed (Figure 41).

\section{Discussion}

Among the major problems of colon cancer are, on the one hand, the late diagnosis in the metastatic stage (one out of five patients had LM at the time of diagnosis) (11) and on the other hand the high risk of recurrence even after surgical therapy and with adjuvant systemic therapies $(70 \%$ recurrence) (2). A better stratification of patients may be useful in improving prognosis and evolution of patients with LM of colon cancer.

HGP may be useful for this purpose. It was demonstrated that pushing GP had an adverse impact on overall survival and disease-free survival (12). Studies have suggested that patients with dHGP had a better prognosis compared to patients with non-dHGP $(13,14)$. But the mechanisms of appearance of this connective ring remain questionable. It is not well known if the tumor stimulates fibrogenesis or if the stromal reaction represents a hepatic defense mechanism or another 


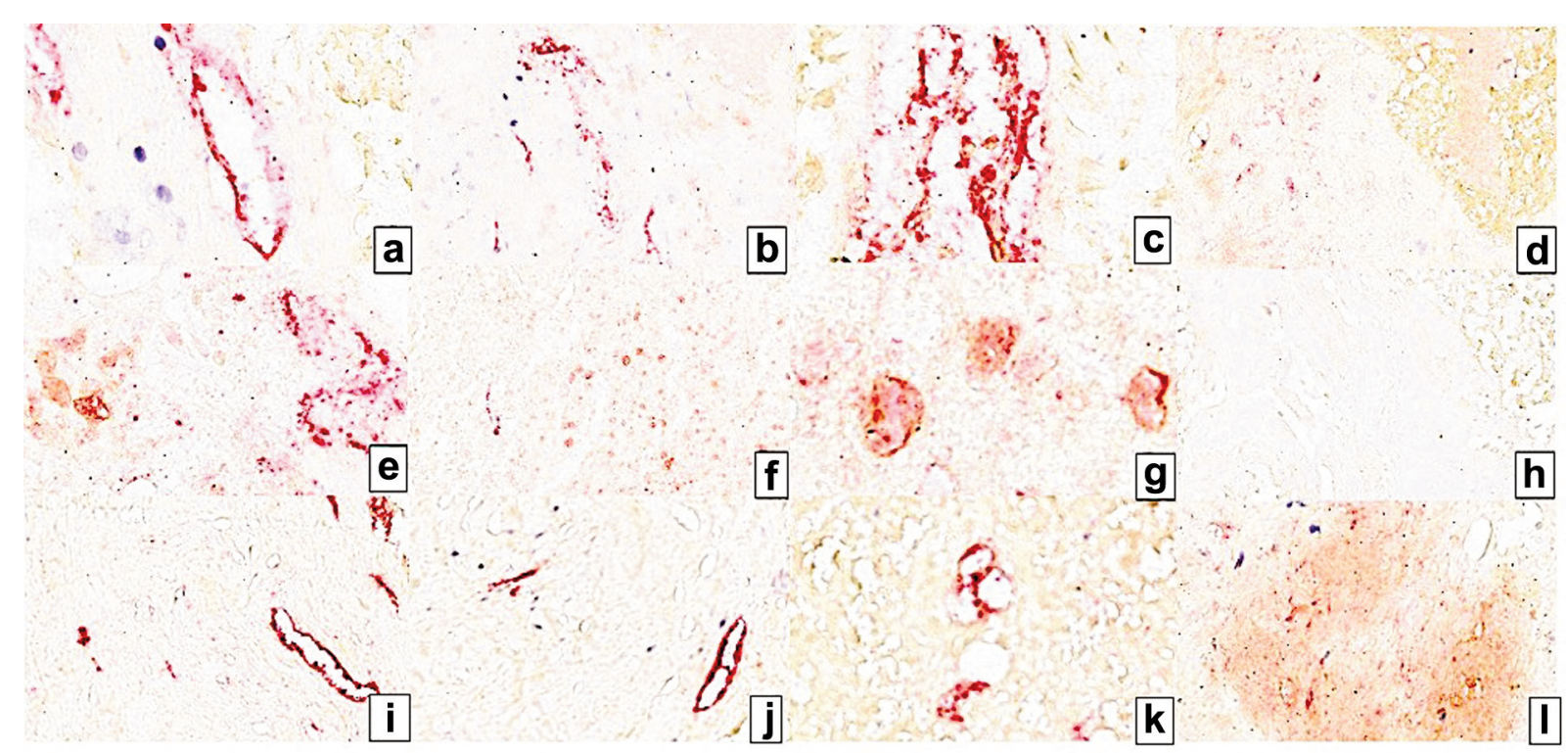

Figure 4. Immunoexpression of CD34/Ki67 and podoplanin/Ki67 on first day after transfer ( $a-c$ and $d$ ), second day after first transfer (e, $f$, $g$ and $h)$ and the fifth day after the second transfer $(i, j, k$ and $l)$.

mechanism. It has been demonstrated that relapse-free survival after hepatectomy was $11.5 \%$ for mature/intermediate desmoplastic reactions (DR) in the liver and $5.6 \%$ for immature desmoplastic reaction (DR) liver (15). One of the most important players in DR formation, producing proteins, such as collagen, extracellular matrix is the cancer associated fibroblasts (CAFs). The metastatic cancer cells pass through the circulation as a single cell or as clusters containing CAFs.

Some of the circulating tumor cells may express epithelial markers (epithelial cell adhesion molecule) and mesenchymal cytokeratin markers (K8, 18, 19). It was demonstrated that the intermediate phenotype had higher plasticity for microenvironment adaptation and a higher aggressiveness and resistance to therapy in breast cancer (16). In our study the K20 immunoexpression decreased during the successive transfer. Keratin 8/18 was expressed in normal (simple epithelium in the liver, pancreas, kidney) and pathological conditions (adenocarcinoma, squamous cell carcinoma) (17). Previous data on dHGP of CRLM indicated isolated or clusters of E-cadherin ${ }^{+} /$keratin $8.18+$ cells (hybrid differentiated phenotype) (18). In the present study a decrease of intensity reaction for E-cadherin and the number of epithelial phenotype cells (E-cadherin ${ }^{+} / \mathrm{vim}^{-} ; \mathrm{K}^{+} / \mathrm{vim}^{-}$) and a slight increase in the number of mesenchymalepithelial differentiated phenotype was noticed after the first transfer. It was associated with decreased levels of podoplanin. The role of podoplanin in modulation of signaling pathways that regulate proliferation, migration, epithelial-mesenchymal transition, is well known.
Another important and more recently described component involved in migration and metastatic process in different types of digestive tumors was CLIC1. CLIC1 activity induced cell-cycle progression and cell division, mainly during $G_{1} / S$ phase in normal conditions. In cancer cells, it favors the acceleration of the growth rate (19). In the present study, the highest value of intensity was found in xenograft cells after the second transfer.

Imaging investigations may be useful to predict the degree of tumor response, but changes can be difficult to differentiate from pretherapeutic appearance. It was noticed that the most common pattern of progression after the initial response to chemotherapy was an increase in tumor size due to growth of peripheral tumor cells (20). The aspect, with higher values of intensity in the peripheral xenografts cells was noticed on slides, on the second day after the first transfer, in our study. After the second transfer, the xenograft cells presented highest intensity values, except for keratin 20 .

Patient-derived xenografts are useful means to underline the molecular features and drug responsiveness of tumors (21). They were used in our study as well. The dHGP and pushing HGP are considered angiogenic types compared to replacement HGP included in the non-angiogenic category. A study which analyzed CD 34 immunoexpression on CAM xenograft of a LM of pancreatic origin (replacement GP) revealed that intussusceptive phenomenon, an adaptation response to stress and hypoxia, was the principal mechanism of new vessel development (22). In our study, on the patient derived xenograft, an increase in vascular density was noted 
after the second transfer and the main mechanisms of angiogenesis were sprouting and intussusception.

\section{Conclusion}

The present experimental model of successive xenografts passaging on CAM mimicked the metastatic process observed in colorectal cancer. Heterogeneous expression of main molecular markers of the CRLM xenograft with dGP on consecutive passages on CAM sustains the presence of a high molecular heterogeneity of metastatic tumor cells from multiple metastases inside the liver. Our findings partially support the therapy response heterogeneity often observed during chemotherapy applied for colorectal cancer.

\section{Conflicts of Interest}

The Authors declare that they have no conflicts of interest.

\section{Authors' Contributions}

ARC designed the study and wrote the paper; AMC and MR evaluated tumor xenografts by microscopy and immunohistochemistry interpretation and validated the manuscript; $\mathrm{AC}, \mathrm{AB}$, and $\mathrm{OMC}$ made surgery for liver metastasis and harvested tissue specimens.

\section{Acknowledgements}

All publication costs are kindly supported by Victor Babes University of Medicine and Pharmacy Timisoara from internal funds allocated for science and research. The Authors are grateful to Patricia Berzava and Ciprian Onica for their excellent technical support.

\section{References}

1 Akgül Ö, Çetinkaya E, Ersöz Ş and Tez M: Role of surgery in colorectal cancer liver metastases. World J Gastroenterol 20(20): 6113-6122, 2014. PMID: 24876733. DOI: 10.3748/wjg.v20. i2 20.6113

2 Zarour LR, Anand S, Billingsley KG, Bisson WH, Cercek A, Clarke MF, Coussens LM, Gast CE, Geltzeiler CB, Hansen L, Kelley KA, Lopez CD, Rana SR, Ruhl R, Tsikitis VL, Vaccaro GM, Wong MH and Mayo SC: Colorectal cancer liver metastasis: Evolving paradigms and future directions. Cell Mol Gastroenterol Hepatol 3(2): 163-173, 2017. PMID: 28275683. DOI: 10.1016/j.jcmgh.2017.01.006

3 Lopez G, Boggio F, Ferrero S, Fusco N and Del Gobbo A: Molecular and immunohistochemical markers with prognostic and predictive significance in liver metastases from colorectal carcinoma. Int J Mol Sci 19(10): 3014, 2018. PMID: 30282914. DOI: $10.3390 / \mathrm{ijms} 19103014$

4 Nordlinger B, Sorbye H, Glimelius B, Poston GJ, Schlag PM, Rougier P, Bechstein WO, Primrose JN, Walpole ET, Finch-Jones M, Jaeck D, Mirza D, Parks RW, Mauer M, Tanis E, Van Cutsem E, Scheithauer W, Gruenberger T, EORTC Gastro-Intestinal Tract Cancer Group, Cancer Research UK, Arbeitsgruppe Lebermetastasen und-tumoren in der Chirurgischen Arbeitsgemeinschaft Onkologie (ALM-CAO), Australasian Gastro-Intestinal Trials Group (AGITG) and Fédération Francophone de Cancérologie Digestive (FFCD): Perioperative FOLFOX4 chemotherapy and surgery versus surgery alone for resectable liver metastases from colorectal cancer (EORTC 40983): long-term results of a randomised, controlled, phase 3 trial. Lancet Oncol 14(12): 1208-1215, 2013. PMID: 24120480. DOI: 10.1016/S1470-2045(13)70447-9

5 Barnhill R, Vermeulen P, Daelemans S, van Dam PJ, RomanRoman S, Servois V, Hurbain I, Gardrat S, Raposa G, Nicolas A, Dendale R, Pierron G, Desjardins L, Cassoux N, PipernoNeumann S, Mariani $\mathrm{P}$ and Lugassy C: Replacement and desmoplastic histopathological growth patterns: A pilot study of prediction of outcome in patients with uveal melanoma liver metastases. J Pathol Clin Res 4(4): 227-240, 2018. PMID: 29917326. DOI: $10.1002 / \mathrm{cjp} 2.105$

6 Temido MJ, Caetano Oliveira R, Martins R, Serôdio M, Costa B, Carvalho C, Santos E, Ferreira L, Teixeira P, Cipriano MA, Tralhão JG and Alexandrino $\mathrm{H}$ : Prognostic factors after hepatectomy for gastric adenocarcinoma liver metastases: Desmoplastic growth pattern as the key to improved overall survival. Cancer Manag Res 12: 11689-11699, 2020. PMID: 33244263. DOI: $10.2147 / C M A R . S 264586$

7 Stessels F, Van den Eynden G, Van der Auwera I, Salgado R, Van den Heuvel E, Harris AL, Jackson DG, Colpaert CG, van Marck EA, Dirix LY and Vermeulen PB: Breast adenocarcinoma liver metastases, in contrast to colorectal cancer liver metastases, display a non-angiogenic growth pattern that preserves the stroma and lacks hypoxia. Br J Cancer 90(7): 1429-1436, 2004. PMID: 15054467. DOI: 10.1038/sj.bjc.6601727

8 van Dam PJ, van der Stok EP, Teuwen LA, Van den Eynden GG, Illemann M, Frentzas S, Majeed AW, Eefsen RL, Coebergh van den Braak RRJ, Lazaris A, Fernandez MC, Galjart B, Laerum OD, Rayes R, Grünhagen DJ, Van de Paer M, Sucaet Y, Mudhar HS, Schvimer M, Nyström H, Kockx M, Bird NC, Vidal-Vanaclocha F, Metrakos P, Simoneau E, Verhoef C, Dirix LY, Van Laere S, Gao ZH, Brodt P, Reynolds AR and Vermeulen PB: International consensus guidelines for scoring the histopathological growth patterns of liver metastasis. Br J Cancer 117(10): 1427-1441, 2017. PMID: 28982110. DOI: 10.1038/bjc.2017.334

9 Stremitzer S, Vermeulen P, Graver S, Kockx M, Dirix L, Yang D, Zhang W, Stift J, Wrba F, Gruenberger T, Lenz HJ and Scherer SJ: Immune phenotype and histopathological growth pattern in patients with colorectal liver metastases. Br J Cancer 122(10): 1518-1524, 2020. PMID: 32205863. DOI: 10.1038/s41416-020-0812-Z

10 van Dam PJ, Daelemans S, Ross E, Waumans Y, Van Laere S, Latacz E, Van Steen R, De Pooter C, Kockx M, Dirix L and Vermeulen PB: Histopathological growth patterns as a candidate biomarker for immunomodulatory therapy. Semin Cancer Biol 52(Pt 2): 86-93, 2018. PMID: 29355613. DOI: 10.1016/ j.semcancer.2018.01.009

11 Mao Y, Chen B, Wang H, Zhang Y, Yi X, Liao W and Zhao L: Diagnostic performance of magnetic resonance imaging for colorectal liver metastasis: A systematic review and metaanalysis. Sci Rep 10(1): 1969, 2020. PMID: 32029809. DOI: 10.1038/s41598-020-58855-1

12 Falcão D, Alexandrino H, Caetano Oliveira R, Martins J, Ferreira L, Martins R, Serôdio M, Martins M, Tralhão JG, Cipriano MA and Castro E Sousa F: Histopathologic patterns as markers of 
prognosis in patients undergoing hepatectomy for colorecta cancer liver metastases - Pushing growth as an independent risk factor for decreased survival. Eur J Surg Oncol 44(8): 1212-1219, 2018. PMID: 29691114. DOI: 10.1016/j.ejso.2018.03.023

13 Galjart B, Nierop PMH, van der Stok EP, van den Braak RRJC, Höppener DJ, Daelemans S, Dirix LY, Verhoef C, Vermeulen PB and Grünhagen DJ: Angiogenic desmoplastic histopathological growth pattern as a prognostic marker of good outcome in patients with colorectal liver metastases. Angiogenesis 22(2): 355-368, 2019. PMID: 30637550. DOI: 10.1007/s10456-019-09661-5

14 Kow AWC: Hepatic metastasis from colorectal cancer. J Gastrointest Oncol 10(6): 1274-1298, 2019. PMID: 31949948. DOI: $10.21037 /$ jgo.2019.08.06

15 Ao T, Kajiwara Y, Yonemura K, Shinto E, Mochizuki S, Okamoto $\mathrm{K}$, Kishi $\mathrm{Y}$ and Ueno $\mathrm{H}$ : Morphological consistency of desmoplastic reactions between the primary colorectal cancer lesion and associated metastatic lesions. Virchows Arch 477(1): 4755, 2020. PMID: 31932918. DOI: 10.1007/s00428-019-02742-2

16 Gonzalez-Villarreal CA, Quiroz-Reyes AG, Islas JF and GarzaTreviño EN: Colorectal cancer stem cells in the progression to liver metastasis. Front Oncol 10: 1511, 2020. PMID: 32974184. DOI: $10.3389 /$ fonc. 2020.01511

17 Galarneau L, Loranger A, Gilbert S and Marceau N: Keratins modulate hepatic cell adhesion, size and G1/S transition. Exp Cell Res 313(1): 179-194, 2007. PMID: 17112511. DOI: 10.1016/j.yexcr.2006.10.007

18 Ceausu AR, Ciolofan A, Cimpean AM, Magheti A, Mederle O and Raica M: The mesenchymal-epithelial and epithelialmesenchymal cellular plasticity of liver metastases with digestive origin. Anticancer Res 38(2): 811-816, 2018. PMID: 29374706. DOI: 10.21873 /anticanres. 12288
19 Barbieri F, Verduci I, Carlini V, Zona G, Pagano A, Mazzanti M and Florio T: Repurposed biguanide drugs in glioblastoma exert antiproliferative effects via the inhibition of intracellular chloride channel 1 activity. Front Oncol 9: 135, 2019. PMID: 30918838. DOI: $10.3389 /$ fonc.2019.00135

20 Paulatto L, Dioguardi Burgio M, Sartoris R, Beaufrère A, Cauchy F, Paradis V, Vilgrain V and Ronot M: Colorectal liver metastases: radiopathological correlation. Insights Imaging 11(1): 99, 2020. PMID: 32844319. DOI: 10.1186/s13244-02000904-4

21 DeBord LC, Pathak RR, Villaneuva M, Liu HC, Harrington DA, Yu W, Lewis MT and Sikora AG: The chick chorioallantoic membrane (CAM) as a versatile patient-derived xenograft (PDX) platform for precision medicine and preclinical research. Am J Cancer Res 8(8): 1642-1660, 2018. PMID: 30210932.

22 Ciolofan A, Creţu OM, Mogoantă SŞ and Ciucă EM: The tyrosine kinase inhibitors effects on metastatic tumor graft in the chick chorioallantoic membrane assay. Rom J Morphol Embryol 58(4): 1257-1262, 2017. PMID: 29556614.
Received June 6, 2021

Revised July 11, 2021

Accepted July 15, 2021 